\title{
La Antropología Forense en España desde la perspectiva de la medicina forense.
}

\author{
Forensic anthropology in Spain from the forensic medicine \\ perspective.
}

\section{JL. Prieto 1}

\section{RESUMEN}

La Antropología forense se ha desarrollado en el mundo de forma diferente en respuesta a criterios específicos de cada país, especialmente en función de consideraciones históricas relativas al enfoque de las investigaciones forenses, su organización profesional, el papel que el antropólogo forense juega en ellas y los sistemas de formación existentes.

En España, los problemas asociados a la identificación en el ámbito legal han estado invariablemente asociados a la Medicina legal y Forense cuyo origen, en su etapa moderna, coincide con el de la propia Antropología, en la que médicos y naturalistas han tenido un impacto significativo.

A pesar del camino recorrido, la Antropología forense es una disciplina en desarrollo en España, como se evidencia por el incremento de la demanda formativa y de los textos y artículos publicados, cada vez con mayor frecuencia en los últimos años.

Palabras clave: Antropología forense, Historia, España, Medicina legal, Medicina forense.

\section{ABSTRACT}

Forensic anthropology has developed in different ways across the world in response to specific country criteria in approaches to forensic investigations, the role that the forensic anthropologist plays in these investigations and their background and/or the type of training system implemented.

In Spain, identification when dealing with living subjects and that of the deceased in the legal sphere, is an activity clearly linked to legal and forensic medicine, usually practised inside the organisational system of forensic medicine. Otherwise, the origin of modern forensic medicine and anthropology, on which doctors and naturalists had a significant impact, is coincident in time.

In spite of the way covered, forensic anthropology is a growing scientific discipline in Spain, as evidenced by the increase in specific courses, texts and articles published in national and international journals, becoming more and more frequently in recent years.

Key words: Forensic anthropology, History, Spain, Legal Medicine. Forensic Medicine, Identification.

Correspondencia: Dr. José Luis Prieto. E-mail: prietoaf@med.ucm.es.

${ }^{1}$ Médico Forense. Especialista en Antropología y Odontología Forense. Profesor Asociado de la Escuela de Medicina Legal de Madrid. 


\section{INTRODUCCIÓN:}

La Antropología forense se ha desarrollado en el mundo de forma diferente en respuesta a criterios específicos de cada país, especialmente en función de consideraciones históricas relativas al enfoque de las investigaciones forenses, su organización profesional, el papel que el antropólogo forense juega en ellas y los sistemas de formación existentes (Baccino y cols. 2004; Brickley y Ferlini 2006; Gulec e İşcan 1994; İşcan 1998; 2001; İşcan y Quatrehomme 1999; İşcan y Olivera 2000; Rodríguez 2004; Sanabria 2004; Schiwy-Bochat y cols. 2004; Ubelaker 1996).

En algunos países, particularmente en los Estados Unidos y bajo su influjo en la mayoría de países de América Latina, la Antropología forense se ha desarrollado desde el concepto de las denominadas "Ciencias forenses", como una subdisciplina de la Antropología física aplicada a la resolución de casos criminales y se practica por antropólogos especializados en Antropología física, o bioantropólogos, con formación en el campo forense. Sus investigaciones y técnicas se aplican con el objetivo de proporcionar datos para la identificación de los restos humanos tales como el sexo, la edad, el origen ancestral o la estatura, es decir, el conjunto de datos que configuran el denominado "perfil biológico" del individuo (Hunter 1996; Krogman e İşcan 1986; Stewart 1979; Ubelaker 1999).

En contraste a este modelo, en los países europeos, especialmente aquéllos de raíz latina, lo que actualmente denominamos como Antropología forense ha estado ligado desde sus inicios a la Medicina forense, desarrollándose en la práctica como una especialidad de esta disciplina, siendo por esta razón más frecuente que la Antropología forense sea practicada por médicos legistas o forenses con formación especíica en antropología física (Baccino y cols. 2004; Gulec e Işcan 1994; Işcan y Quatrehomme 1999; Prieto 2006).

\section{RAÍCES HISTÓRICAS: ANTROPOLOGÍA Y MEDICINA LEGAL EN ESPAÑA: Período Clásico.-}

En España, los problemas asociados a la identificación en el ámbito legal han estado siempre asociados a la Medicina Legal y Forense (Etxeberría 2004; Prieto 200 I; Reverte 199I; Sánchez 1996), siendo uno de los temas abordados en los textos clásicos de la especialidad (Aznar y Maestre 1945; Ballesteros 1913; Barahona 1908; Gisbert Calabuig 1985; Lecha Martínez 1894; López Gómez y Gisbert Calabuig 1967; Mata 1874; Peiró y Rodrigo I844; Piga 1928; Royo-Villanova 1952).

Las raíces históricas de la Antropología forense se remontan a mediados del siglo XIX, bajo la influencia de dos principales eventos: (I) la constitución de la Société d'anthropologie du Paris, por el Professor Paul Broca y (2) el desarrollo de la moderna Medicina legal, muy influenciada por la Antropología. Médicos y naturalistas han tenido un impacto significativo en el desarrollo de la Antropología en España. Una de sus principales figuras fue el Dr. Pedro González de Velasco, anatomista fundador de la Sociedad Antropológica Española en 1865 y del Museo Antropológico de Madrid en 1875, fechas en la que tienen su aparición las primeras publicaciones sobre Antropología, tales como la Revista de Antropología en I 874 y Antropología Moderna en I883 (Reverte 1991).

En 1883, se crearon las secciones de Antropología y Etnografía del Museo de Antropología, a lo que siguió en 1892 la designación del profesor Manuel Antón y Ferrandis como primer catedrático de Antropología de la Facultad de Ciencias de la Universidad Central de Madrid. En 1910 Ferrandis fue nombrado primer director del Museo de Antropología, Etnografía y Prehistoria. Otros eventos significativos fueron la publicación en 1883 del Índice Cefálico en España por el Dr. Federico Olóriz Aguilera, catedrático de Anatomía de la Universidad de Madrid (Olóriz 1894) y la creación 
del Museo Craneológico que llegó a reunir un total de 2500 cráneos, 2220 de ellos correspondientes a individuos identificados (Gómez Ocaña 19|3). Otras importantes investigaciones realizadas por Olóriz incluyen su publicación sobre la estatura en España (Olóriz 1886). Desgraciadamente, la falta de colaboración para la recopilación de datos de algunas regiones españolas motivó el abandono de Olóriz de dicho proyecto. Su interés en las técnicas de identificación se volcó entonces en las huellas dactilares, de las que es uno de los fundadores, debiéndose a él el desarrollo del denominado "sistema monodactilar" o "español" (Olóriz 1908, 1909, 1910, 1911).

De finales del siglo XIX y comienzos del XX destacan las publicaciones de otros ilustres fundadores de la Antropología española, como Telésforo de Aranzadi, catedrático de Antropología de la Universidad de Barcelona y Luis de Hoyos Sáinz, profesor de Fisiología y de la Escuela Superior de Magisterio de Madrid (Aranzadi y Hoyos Sáinz 1917; Hoyos Sáinz y Aranzadi 1913; Hoyos Sáinz 1893, 1939).

En el mismo periodo la Antropología juega un papel protagonista en tres áreas de la Medicina legal: ( I el análisis de la relación entre los rasgos físicos y la conducta criminal, la denominada Antropología criminal, (2) el establecimiento de la identidad de individuos vivos (especialmente delincuentes) con el propósito de facilitar las identificaciones policiales, a través de la Somatometría o Antropología Judicial y (3) el establecimiento de la identidad de un cadáver, particularmente en el caso de restos humanos, cadáveres mutilados, quemados y/o esqueletizados, en los que se hace necesario establecer el perfil biológico (sexo, edad, estatura, etc).

La Antropología Criminal se hizo famosa gracias a los trabajos de César Lombroso, quien consideraba a los delincuentes una subespecie anormal de la raza humana. Para Lombroso, el criminal desarrolla una conducta innata que representa una regresión a estados evolutivos previos y que se puede reconocer por una serie de estigmas o anomalías tales como la asimetría craneal o facial, una frente corta, arcos superciliares prominentes, prognatismo mandibular, una implantación irregular del pelo y los dientes y lo que él considera la característica más atávica de los criminales, un hoyuelo en mitad del occipital. Desde el punto de vista psicológico, predominan los instintos primitivos con una incapacidad congénita para resistir las fuerzas del mal (Lombroso 1897). En España, uno de sus representantes más destacados es Rafael Salillas (Salillas 1888, 1908). Otros famosos psiquiatras de la época también incorporaron las teorías antropológicas en sus intervenciones en los tribunales. Los doctores Ángel Pulido Fernández, José María Esquerdo Zaragoza, Luis Simarro Lacabra y Jaime Vera López, actuaron con frecuencia en procesos judiciales realizando evaluaciones científicas de comportamientos criminales (Fernández |99|).

El método antropométrico, presentado por Alphonse Bertillon en el Congreso Médico de Roma de 1882 (Barahona 1908), tenía como meta la identificación de criminales reincidentes tomando como base la casi absoluta invariabilidad del esqueleto humano adulto, la variabilidad interindividual de las medidas esqueléticas y su simplicidad y precisión en sujetos vivos, proporcionando una clasificación práctica, sencilla y exacta. Este método fue rápidamente adoptado por España, contribuyendo a su difusión los trabajos de antropólogos físicos españoles tales como Álvarez Taladriz, García Plaza, Alonso y Aranzadi (Lecha Martínez 19|2). El ulterior descubrimiento de las huellas dactilares, a partir de los trabajos de Vucetich y especialmente de Olóriz en España, supuso el fin de las técnicas de identificación antropométrica de Bertillon. Sin embargo, las técnicas somatométricas han regresado de nuevo en los casos de identificación de delincuentes a través de imágenes obtenidas por videocámaras (Ventura y cols. 2004; Porter y Doran 2000).

Una figura trascendental de la Medicina legal en España es el Dr. Pedro Mata Fontanet. En 1843 se crea la primera cátedra de Medicina legal en la Universidad de Madrid, al frente de la cual 
se nombra al Dr. Mata. A él le debemos la creación en 1862 de un cuerpo de médicos del Estado dependientes del Ministerio de Justicia, al servicio de Juzgados y Tribunales, que será denominado Cuerpo Nacional de Médicos Forenses, cuyo título y funciones se encuentran reconocidas oficialmente en la Ley de Enjuiciamiento Criminal de I 882. En este contexto, la Antropología es reconocida como una materia principal de la Medicina forense. Del primer programa oficial de oposiciones para acceso al Cuerpo, del año 1915, 28 temas corresponden a los capítulos de antropometría, craneometría y características esqueléticas relativas al sexo y edad, o el desarrollo dental (Vibert 1916). El trabajo de antropólogos españoles como los anteriormente mencionados Olóriz o Aranzadi son citados en los textos de Medicina legal de la época (Barahona 1908; Lecha Marzo 1917; Piga 1928) describiéndose en los mismos las técnicas antropológicas de identificación del cadáver. En ellos encontramos referencias y descripciones antropométricas referentes al diagnóstico de la estatura (tablas de Orfila, Rollet o Manouvrier); sexo; edad (puntos de osificación, cierre de suturas, osificación laríngea, estado de los dientes) (Barahona 1908; Lecha Martínez 1912; Peiró y Rodrigo I84I; Piga 1928); marcas particulares (fracturas antiguas, modificaciones ocupacionales) así como al uso de los rayos X (Barahona 1908) y al análisis dental (Piga 1928). Algunos autores como Lecha Marzo (1917) recogen la importancia del análisis microscópico del hueso en la estimación de la edad y de la data de la muerte, a partir de estudios previos desarrollados por Tirelli. Los textos de Maestre (Ballesteros 1913) primer director del Instituto de Medicina Legal, Toxicología y Psiquiatría de España, creado en 1914 o Piga (1928, 1935) sucesor de Maestre en la Cátedra de Medicina Legal de Madrid, son particularmente minuciosos al abordar las cuestiones relativas a la identificación del cadáver y, aunque en su mayoría presentan los datos de trabajos desarrollados por otros autores, aportan valiosos testimonios de su experiencia personal.

El periodo comprendido entre la segunda mitad del siglo XIX y la Guerra Civil Española ( 1936 - 1939) es conocido como la "Edad de Plata" de la cultura y la ciencia españolas. En esta época, instituciones como el Ateneo de Madrid y particularmente la Institución Libre de Enseñanza, disfrutan de gran influencia en los ámbitos cultural y cientíico.

La Institución Libre de Enseñanza se funda en 1876 por un grupo de profesores universitarios (Francisco Giner de los Ríos, Gumersindo de Azcárate y Nicolás Salmerón), apartados de la universidad en defensa de la libertad de cátedra. Esta institución, con la que colaboraron la mayor parte de los más prestigiosos cientíícos españoles del momento, marcó un periodo significativo del desarrollo de la cultura científica española. Uno de sus principales logros fue la creación de la Junta para Ampliación de Estudios, que estuvo a cargo de la creación del Instituto Nacional de Ciencias Físico-Naturales al que incorporó el Museo de Antropología (al frente del cual estaba Manuel Antón y Ferrándiz). El principal propósito de este museo fue introducir en España las teorías pedagógicas y científicas que se estaban desarrollando internacionalmente. Desgraciadamente, la Guerra Civil provocó la destrucción de esta institución y con ello, el incipiente sistema científico que había comenzado en España (Otero Carvajal 200 I).

\section{Período Moderno.-}

Mientras que la Antropología forense se desarrollaba rápidamente fuera de España, especialmente en los Estado Unidos, tras la Segunda Guerra Mundial, bajo el impulso de los Drs. Alex Hrdliča, Wilton Marion Krogman Ellis R. Kerley y T.D. Stewart, en nuestro país los avances eran relativamente escasos. Cabe resaltar en este periodo las investigaciones sobre restos esqueléticos con fines forenses, llevadas a cabo por el Dr. Blas Aznar, impulsor de la Criminalística en España (Aznar 1931) y el ya mencionado Tomás Maestre (Aznar y Maestre 1945). Los tratados de 
reputados autores como los Drs. Royo-Villanova (Royo-Villanova 1952), Lopez Gómez (1967) y Gisbert Calabuig (1985) y los trabajos científicos referentes al estudio de restos humanos, continuaron durante décadas haciendo exclusiva referencia a los textos y autores más clásicos de la disciplina y a conocimientos desactualizados (Martínez Estrada, 195I, 1952; Muñoz Tuero y de Portugal Álvarez, 1966; Muñoz Tuero, Moya Pueyo y Villalaín Blanco, 1972; Muñoz Tuero y Díaz Domínguez, 1981; Pérez de Petinto, 1952, 1980; Romero Palanco 1980; Serrano Cepedano, 1982; Villalaín Blanco y Buján Varela I981; Villalaín Blanco y Ramos Almazán 198I).

A principios de los años 80 comienza un nuevo periodo para la Antropología forense española, cuyo punto de referencia es la creación del Laboratorio de Antropología Forense y Paleopatología de la Escuela de Medicina Legal de Madrid, a cargo del Prof. José Manuel Reverte Coma (Reverte 1997). Con sus luces y sus sombras, el profesor Reverte fue responsable de crear un grupo, principalmente formado por médicos legistas o forenses, interesado en la disciplina. A través de la publicación de su libro Antropología Forense (Reverte Coma 1991), el primero en España sobre la materia, las técnicas y conocimientos desarrollados por la Antropología forense norteamericana se difunden en nuestro país. Este resurgimiento renovado de las técnicas de estudio de restos humanos, bajo la denominación de Antropología forense, coincide con varios acontecimientos substanciales que van a marcar en el futuro el rumbo de la Antropología forense en España. Principalmente la fundación en el año 1986 de la Asociación Española de Paleopatología y, muy especialmente, el comienzo de la idea de modernización de la Medicina legal en España, con la reimplantación en 1984 de la Especialidad de Medicina Legal y Forense (en cuyo programa de formación se incluye la Antropología forense) y la publicación en el año 1985 de la Ley Orgánica del Poder Judicial que desarrolla la figura de los Institutos de Medicina Legal. De esta forma, la Antropología forense se incorpora en nuestro país al curriculum de Medicina legal y comienza a aplicarse, en su concepción moderna, en la práctica médico forense cotidiana.

En el año 1985, la legislación reconoce, en la mencionada Ley Orgánica del Poder Judicial, la necesidad de transformar el sistema organizativo de la Medicina forense en España, a través del desarrollo de los denominados Institutos de Medicina Legal. Los Institutos nacen con el ánimo de promover la modernización de la Medicina forense, fomentando el trabajo en equipo y la especialización, en centros equipados adecuadamente.

Durante I 5 años, el Instituto Anatómico Forense de Madrid, dirigido por el Dr. José María Abenza Rojo, trabajó en el desarrollo del embrión que debería llegar a ser una parte importante del futuro Instituto de Medicina Legal, llevándose a cabo en este periodo la creación y consolidación de servicios de Anatomía Patológica, Toxicología, Radiología y Antropología y Odontología forenses, constituyendo éste último el primer laboratorio de su especialidad creado (en el año 1992) en el seno del Cuerpo Nacional de Médicos Forenses. Esta estructura se plasmó en el Reglamento del Instituto de Medicina Legal de Madrid, publicado en el año 2006 (Decreto 37/2006, de 4 de mayo, por el que se crea el Instituto de Medicina Legal de la Comunidad de Madrid y se aprueba su Reglamento. BOCM n 109, de 9 de mayo) en el que se contempla una Sección de Antropología y Odontología Forense, encuadrada en el Servicio de Patología, encargada de: "la identificación de sujetos vivos y cadáveres, así como el estudio de los restos humanos para su identificación y el establecimiento de la causa y circunstancias de la muerte".

Desgraciadamente, diversas circunstancias que no han de ser motivo de análisis en este trabajo, entre ellas la aún pendiente puesta en marcha del Instituto de Medicina Legal (único que no ha sido creado en España) desembocaron en el año 2008, en el cierre de dicho laboratorio que me honro en haber desarrollado y dirigido. 
El Ministerio de Educación y Ciencia, siguiendo la denominación internacional de la UNESCO, considera la "Antropología Forense" como una subdisciplina (2402.03) de la "Antropología Física" (2402) encuadrada en el campo de las Ciencias de la Vida (24). Como se ha remarcado anteriormente, los problemas de identificación de sujetos vivos y cadáveres en la esfera legal en nuestro país, se han asociado siempre a la Medicina legal y forense (Etxeberría 2004; Prieto 200 I; Reverte 1991; Sánchez 1996) de forma que, en la práctica, la Antropología forense constituye una auténtica especialidad de la Medicina legal y forense cuyo propósito es el estudio médico legal, en especial de los restos humanos, para su identificación y el establecimiento de la causa y circunstancias de la muerte (Prieto $200 \mathrm{I}$ ). Estos dos últimos elementos son fundamentales en la resolución de cualquier caso de muerte en un contexto judicial y constituyen objetivos indiscutibles de cualquier autopsia médico legal (como estipula la Ley de Enjuiciamiento Criminal) cualquiera que sea la condición en que se encuentra el cuerpo o su estado de conservación. En este sentido, la Unión Europea, en la Recomendación 99(3) del Consejo de Ministros de los Estados Miembros para la Armonización de las Autopsias Médico Legales (1999) establece en los Principios y Reglas de los procedimientos de autopsia médico-legal, que éstas "deben ser practicadas en todos los casos evidentes o sospechosos de muerte no natural" $y$, en particular, "en los casos de cuerpos no identificados o esqueletizados", consideración sobre la que abunda el Manual de las Naciones Unidas sobre la Prevención e Investigación Eficaces de las Ejecuciones Extralegales, Arbitrarias o Sumarias, o Protocolo Minnesota (199|).

En este contexto, cabe resaltar el hecho de que el experto en Antropología forense juega cada día un papel más relevante en el estudio de cadáveres frescos sometidos a autopsias convencionales, contribuyendo al diagnóstico de la causa y especialmente de las circunstancias de la muerte, complementando el trabajo del médico o patólogo forense. Nuestro trabajo es especialmente útil a la hora de proporcionar datos adicionales sobre las características de lesiones (traumatismos contusos, lesiones por arma blanca y de fuego) y los objetos responsables de las mismas, cuando éstas afectan a estructuras óseas. Un antropólogo forense que trabaja en los centros en los que se practican diariamente las autopsias de cadáveres judiciales (Institutos de Medicina Legal) adquiere una experiencia inestimable a la hora de aplicar sus conocimientos en el diagnóstico de lesiones sobre restos esqueléticos y aporta una perspectiva diferente a la de los médicos o patólogos forenses sin formación específica en el análisis de las estructuras óseas. Por otra parte, la necesaria vinculación de estos centros a la Universidad ha de facilitar que la práctica, la docencia y la investigación vayan definitivamente de la mano.

Los principales centros que cuentan en la actualidad con laboratorios de Antropología forense al servicio de la Administración de Justicia son los siguientes:

- Institutos de Medicina Legal (Cataluña y Galicia).

- Instituto Nacional de Toxicología y Ciencias Forenses (Departamento de Madrid).

- Departamentos Universitarios, principalmente de Medicina legal (Madrid, País Vasco, Valencia, Alicante y Granada).

- Áreas de Policía Científica de los Cuerpos Nacionales de Policía (Policía Nacional y Guardia Civil).

Aunque no existen estadísticas que permitan conocer el número total de casos forenses (judiciales) investigados anualmente por estos laboratorios, un cálculo aproximado sitúa esta cifra en unos 200 (dato derivado de una pequeña encuesta personal llevada a cabo hace dos años). Esta cantidad es a todas luces muy inferior al número de casos que objetivamente requerirían un estudio antropológico, coincidiendo con lo observado en otros países (Cattaneo y Baccino 2002). Sirva 
como ejemplo el caso del laboratorio del Instituto Anatómico Forense de Madrid. De una media anual en torno a los 2500 cadáveres, la demanda de estudios antropológicos para Madrid (el laboratorio daba cobertura a Juzgados de toda España) oscilaba entre los 35 y los 40 casos. El desarrollo de la especialidad en los Institutos de Medicina Legal debería facilitar una actuación protocolizada, con criterios de estudio más objetivos y homogéneos, evitando los actuales criterios puramente discrecionales por parte del médico forense a cargo de la autopsia.

\section{ENSEÑANZA:}

\section{Formación Universitaria.-}

\section{ESTUDIOS DE PREGRADO.}

En la licenciatura de Medicina, algunos temas de la asignatura de Medicina Legal incorporan conceptos relacionados con la Antropología forense, especialmente en relación al estudio de cuerpos carbonizados, las técnicas de identificación, descomposición cadavérica y actuación en desastres.

La universidad del País Vasco en su programa de pregrado incluye una asignatura opcional denominada "Identidad e Identificación Antropológica". Por su parte, la Universidad de Navarra incluye de forma específica la Antropología forense como parte de su lista de asignaturas y la Universidad de Alicante bajo el epígrafe de Antropología Criminológica.

\section{ESTUDIOS DE POSTGRADO.}

\section{Especialidad de Medicina Legal y Forense}

La Antropología forense se encuentra desarrollada dentro del área de Criminología en relación a los aspectos de identificación.

\section{Doctorado}

El único curso específico de Medicina Legal y Forense es el ofertado por la Universidad Complutense de Madrid (Departamento de Toxicología y Legislación Sanitaria). Este curso incluye la Antropología forense en su parte teórica y como materia de investigación para tesis doctorales.

La Universidad Miguel Hernández de Alicante incluye la asignatura denominada Antropología forense, policía científica y dactiloscopia en su curso de doctorado en Medicina Legal.

Otras facultades de Medicina y Ciencias Biológicas ofrecen cursos de doctorado que incluyen temas más o menos relacionados con la Antropología forense.

\section{Cursos Específicos sobre Antropología Forense \\ - Cursos Básicos de Antropología Forense:}

Hoy en día hay una amplia oferta de cursos en las universidades españolas. La Universidad Complutense de Madrid imparte, una vez por año, un curso básico (virtual) de 60 horas lectivas, dirigido a licenciados en Medicina, Derecho, Periodismo, Historia y Arqueología, Biología, Ciencias de la Salud y Criminología, entre otros.

Otras universidades, como la Autónoma de Barcelona, La Universidad Miguel Hernández de Alicante, la Universidad de Granada y el Instituto Canario de Bioantropología y Paleopatología de la Universidad de la Laguna, organizan cursos específicos con mayor o menor regularidad o incluyen temas relacionados con la Antropología forense en cursos de Paleopatología.

- Cursos Master y de Especialidad:

Desde 1997, la Escuela de Medicina Legal de Madrid ofrece una especialidad de 
Antropología forense de 300 horas lectivas, desarrolladas a lo largo de un año académico, dirigido a licenciados con al menos cuatro años de experiencia en el campo.

En el año 2003, la Universidad de Granada inauguró dos cursos virtuales: el Curso de Especialización en Técnicas Antropológicas de Identificación Humana de 70 horas lectivas y el Master Virtual en Antropología Forense y Genética Humana que incluye 260 horas lectivas de Antropología forense.

La Universidad de Valencia organiza un Master en Medicina Forense para médicos, biólogos y odontólogos que incluye una unidad de Antropología forense.

\section{Formación EN EL CUERPO de MÉdICOS Forenses}

El temario de oposiciones al Cuerpo Nacional de Médicos Forenses incluye seis temas sobre identificación, tres de los cuales versan sobre Antropología forense.

El curso de formación inicial, realizado en el Centro de Estudios Jurídicos, una vez superada la oposición, ofrece un temario básico de 10 horas de seminarios en Antropología forense.

Por otra parte, dentro del programa de formación continuada para médicos forenses del Centro de Estudios Jurídicos, se ha venido ofertando irregularmente, desde el año 1997, un Curso Básico en Antropología Forense, con la asistencia del Prof. Douglas Ubelaker, de la Smithsonian Institution.

\section{INVESTIGACIÓN:}

El desarrollo de proyectos de investigación en este campo es relativamente pequeño en España, a lo que ha contribuido especialmente la carencia de colecciones contemporáneas de esqueletos, realizándose la mayoría de las investigaciones sobre colecciones arqueológicas, en el terreno de la Antropología física, con aplicación posterior a cuestiones de índole legal.

La mayor parte del trabajo de investigación que se lleva a cabo en los departamentos universitarios asociados al campo de la enseñanza de la Medicina legal y forense, suele asociarse con la formación de doctorado. Los principales centros de investigación en este campo son aquéllos que llevan a cabo estudios para la Administración de Justicia: universidades Complutense de Madrid, Granada, País Vasco, Valencia, Autónoma de Barcelona, Alicante y Zaragoza.

Las becas y ayudas estatales para la investigación en el campo de la Biomedicina no incluyen a la Medicina forense como área específica y mucho menos a la Antropología forense, por lo que los proyectos presentados habitualmente han de competir en áreas de investigación pura, con equipos muy poderosos, no teniendo prácticamente posibilidades de éxito.

Con estos antecedentes cabe imaginarse que el desarrollo de proyectos en esta área es realmente difícil y en muchas ocasiones éstos deben financiarse por los propios investigadores.

Por otra parte, se está produciendo un incremento en la investigación llevada a cabo en los recientemente creados Institutos de Medicina Legal, lo que se refleja en el número de trabajos científicas publicados en revistas nacionales e internacionales.

\section{Publicaciones sobre Antropología forense en España.-}

Tras la publicación del primer texto del profesor Reverte (199| y |999), se han publicado varias monografías sobre el tema tales como "Identificación Antropológica Policial y Forense" (Villalain y Puchalt 2000) y "Antropología Criminológica" (Rodes y Martí 200 I).

Otras contribuciones importantes derivadas del campo de la antropología arqueológica incluyen los trabajos de Trancho y colaboradores (1997) y la base de datos bibliográfica sobre 
patología de restos esqueléticos elaborada por Etxeberría http://www.aranzadizientziak.org/old/antropologia/0 It.htm).

\section{PUBLICACIONES NACIONALES.}

Entre los años 1992 a 2006 se publicaron un total de 17 artículos relativos a la Antropología forense (Agudo, Sancho y del Río 1998; Chiarri, Rodes y Martí 2003; del Río y Sánchez 1997; del Río, Sánchez y Prieto 2000, 200 I; Exxeberría 1992; Etxeberria y Carnicero 1998; Miquel y Villalaín 1996; Prieto 1996; Prieto y Abenza 1998; Prieto et al. 2006; Ramírez et al. 2000; Reverte 1997; Rodes 2004; Rodes et al. 2004; Sánchez 1996, 1997) en las cuatro publicaciones relacionadas con la Medicina forense (Revista Española de Medicina Legal (http://www.arrakis.es/ anmf/), Cuadernos de Medicina Forense (http://www.cuadernosdemedicinaforense.es), Boletín Galego de Medicina Legal e Forense y Revista Aragonesa de Ciencias Forenses) uno de ellos constituye un número monográfico que compendia los textos del curso básico para médicos forenses (Prieto y cols. 200 I).

\section{PUBLICACIONES INTERNACIONALES.}

En el mismo periodo (1992-2006) se han publicado unos nueve artículos en las revistas más relevantes del ámbito forense (Bolaños y col. 2003; Garamendi 2005; Lorente y col. 200 I; Martín de las Heras y col. 1999; Muñoz y col. 200 I; Prieto, Magaña y Ubelaker 2004; Prieto y col. 2005; Trancho y col. 1997; Valenzuela y col. 2000).

\section{PERSPECTIVAS DE FUTURO:}

Parece evidente que la especialización en Antropología forense y la acreditación de profesionales y laboratorios es una necesidad, dada la falta de una titulación específica. Con el propósito de velar por el avance de la Antropología forense en Europa, contar con antropólogos adecuadamente formados y laboratorios cualificados, en el año 2003 se crea en Milán la Forensic Anthropology Society of Europe (FASE), bajo el patrocinio de la International Academy of Legal Medicine (IALM), de la que constituye su primera sección. Sin duda, FASE tiene un papel principal que jugar en la evolución de la Antropología forense en Europa. Entre otros, actuar como núcleo de encuentro y foro de discusión de los profesionales de la Antropología forense europea y elaborar recomendaciones para la unificación de criterios diagnósticos y de programas de formación, incluyendo el necesario desarrollo de una certificación europea, de manera similar a la que la American Academy of Forensic Sciences ofrece a los profesionales de los Estados Unidos y Canadá.

A pesar del camino recorrido, la Antropología forense es una disciplina en desarrollo en España, como se evidencia por el incremento de la demanda formativa y de los textos y artículos publicados, cada vez con mayor frecuencia en los últimos años. Reflejo de este interés creciente y también del grado de madurez alcanzado por la disciplina, es la reciente fundación de la Asociación Española de Antropología y Odontología Forense (AEAOF), que en el año 2008 celebró su primera reunión científica, que supuso un éxito rotundo con más de 100 inscritos.

Aunque indudablemente la Antropología forense necesita un impulso en nuestro país, es de esperar que el actual modelo organizativo de la Medicina forense, el progreso de los Institutos de Medicina Legal y una mayor vinculación a la Universidad fortaleciendo las áreas de formación e investigación, permitirán desarrollar el máximo potencial de la disciplina. En este proceso, FASE y AEAOF deberán jugar un papel protagonista y complementario. 


\section{BIBLIOGRAFÍA:}

- Agudo Ordóñez, J., Sancho Ruiz, M., \& P.A. Muñoz del. 1998. Identificación positiva mediante la radiografía de los senos frontales. Revista Española de Medicina Legal 82: 45-47.

- Aranzadi, T. \& L. Hoyos Sainz. 1917. Etnografía: sus bases, sus métodos y aplicaciones a España. Madrid. Biblioteca Corona.

- Aznar, B. 193I. Contribución a la identificación de restos óseos fetales. Madrid. Gráficas Gutenberg.

- Aznar, B. \& T. Maestre. 1945. Identificación de Restos Cadavéricos Oseos. Investigación, 21I: 79-8I.

- Baccino, E., Cattaneo, C., Cunha, E., Prieto, J.L. \& R. Penning. 2004. Organization, Teaching and Research in Forensic Anthropology Across Europe. Plenary Session. Ist FASE Meeting. Frankfurt.

- Ballesteros, S. 1903. Apuntes de Medicina Legal y Toxicología ajustados a las explicaciones del Dr. D. Tomás Maestre Pérez. Madrid. Librería Médica de Vidal.

- Barahona Holgado, I. 1908. Lecciones de Medicina Legal. Salamanca. Marcelino Rodríguez.

- Bolaños, M.V., Moussa, H., Manrique, M.C., \& M.J. Bolaños. 2003. Diographic evaluation of third molar development in Spanish children and young people. Forensic Science International 5;133(3): 212-9.

- Brickley, M.B. \& R. Ferlini. 2007. Forensic anthropology: Developments in two continents, in Brickley, M.B. \& R. Ferlini (eds.) Forensic Anthropology: Case Studies from Europe. 3-18 Springfield, IL: Charles C. Thomas.

- Cattaneo, C. \& E. Baccino. 2002. A call for forensic anthropology in Europe. International Journal of Legal Medicine II6: NI-N2.

- Cattaneo, C., Gigli, F., Lodi, F. \& M. Grandi. 2003. The detection of morphine and codeine in human teeth: An aid in the identification and study of human skeletal remains. Journal of Forensic Odontostomatology $2 \mathrm{I}(\mathrm{I}): \mathrm{I}-\mathrm{S}$.

- Council of Europe. Committee of Ministers. Recommendation No. R (99) 3 of the Committee of Ministers to Member States on the Harmonisation of Medico-Legal Autopsy Rules (Adopted by the Committee of Ministers on 2 February 1999 at the 658th meeting of the Ministers' Deputies), pp. 2-3.

- Chiarri, M., Rodes, F. \& J.B. Martí. 2003. Identificación positiva a partir del estudio de restos óseos en un caso de desaparecido. Boletín Galego de Medicina Legal e Forense. II: 35-40.

- Etxeberría, F. 1992. Aspectos macroscópicos del tejido óseo sometido al efecto de las altas temperaturas. Aportación al estudio de las cremaciones. Revista Española de Medicina Legal 72-73: 159-163.

- Etxeberría, F. \& M.A. Carnicero. 1998. Estudio macroscópico de las fracturas del perimortem en Antropología Forense. Revista Española de Medicina Legal 84-85: 36-44.

- Etxeberria, F. 2004. Panorama Organizativo Sobre Antropología y Patología Forense en España. Algunas Propuestas para el Estudio de Fosas con Restos Humanos de la Guerra Civil Española de 1936, in Silva, E., Esteban, A., Castan, J. \& P. Salvador (eds.) La Memoria de los Olvidados. Un Debate sobre el Silencio de la Represión Franquista: 183-219. Valladolid: Ámbito Ediciones.
- Fernandez, P.T. 1991. La defensa de la sociedad. Cárcel y delincuencia en la España de los siglos XVIII-XIX. Madrid. Alliance ed. p. 268.

- Garamendi, P.M., Landa, M.I., Ballesteros, J. \& M.A. Solano. 2005. Reliability of the methods applied to assess age minority in living subjects around 18 years old. A survey on a Moroccan origin population. Forensic Science International 10;154(I):3 -12.

- Gisbert Calabuig, J.A. 1985. Medicina Legal y Toxicología. Valencia. Fundación García Muñoz, pp.675-82.

- Gómez Ocaña. 1913. Memorias de la Real Sociedad Española de Medicina Natural. 7(5): 343-454.

- Gulec, E.S. \& M.Y. İscan. 1994. Forensic anthropology in Turkey. Forensic Science International 66(I):61-8.

- Hoyos Sainz, L. 1899. Técnica antropológica y antropología física. Madrid. Romo y Fussel. (Imp. del Asilo de Huérfanos del S.C. de Jesús).

- Hoyos Sainz, L., \& T. Aranzadi. 1913. Unidades y constantes de la crania Hispanica. Madrid. Asociación Española para el Progreso de las Ciencias.

- Hoyos Sainz, L. 1929. Una hoja para el estudio de la herencia en el hombre : Grupos sanguíneos y caracteres antropológicos. Madrid. Laboratorio de Antropología Fisiológica.

- Hoyos Sainz, L. 1939. Ficha antropológica para la investigación de herencia. Madrid. [s.n.].

- Hunter, J.R. 1996. A background to forensic archaeology, in Hunter, J., Roberts, C. \& A. Martin (eds.) Studies in Crime: An Introduction to Forensic Archaeology. pp. 7-23. London. Batsford Ltd.

- İscan, M.Y. 1998. Progress in Forensic Anthropology: the 20th Century. Forensic Science International 98(I-2): I-8.

- İşcan, M.Y. \& G. Quatrehomme. 1999. Medicolegal anthropology in France. Forensic Science International I00(I-2): 17-35.

- İscan, M.Y. \& H.E. Olivera. 2000. Forensic anthropology in Latin America. Forensic Science International 109(I): 15-30.

- İscan, M.Y. 200I. Global forensic anthropology in the 21st Century. Editorial. Forensic Science International II7: I-6.

- Krogman, W.M. \& M.Y. İşcan. 1986. The Human Skeleton in Forensic Medicine. Springfield. Charles C. Thomas.

- Lecha-Martínez, L. I894. Elementos de Medicina Legal complementarios a la obra de Hofmann. Valladolid. Hijos de Rodríguez.

- Lecha Martínez, L. 1912. Manual de Medicina Legal. Madrid. Imprenta y Librería de Nicolás Moya.

- Lecha Marzo A. 1917. Tratado de autopsias y embalsamamientos. El diagnóstico Médico Legal en el cadáver. Barcelona. Manuel Marín Editor.

- Lombroso, C. 1897. L'uomo delinquente : in rapporto all'antropologia, alla giurisprudenza ed alle discipline carcerarie. Torino. Fratelli Bocca.

- López Gómez, L. \& J.A. Gisbert Calabuig. 1967. Tratado de Medicina Legal. Valencia. Saber. 
- Lorente, J.A., Entrala, C., Alvarez, J.C., Arce, B., Heinrichs, B., Lorente, M., Carrasco, F., Budowle, B. \& E. Villanueva. 2001. Identification of missing persons: The Spanish "Phoenix" program. Croatian Medical Journal 42(3): 267-70.

- Martin-de las Heras, S., Valenzuela, A., Villanueva, E., Marques, T., Exposito, N. \& J.M. Bohoyo. 1999. Methods for identification of 28 burn victims following a 1996 bus accident in Spain. Journal of Forensic Sciences 44(2):428-31.

- Martínez Estrada, J.M. 195I. La Sinóstosis de los huesos del cráneo. Rev.Med.Leg. 64-65: 291-303.

- Martínez Estrada, J.M. 1952. Determinación de la edad en el cráneo en el niño. Rev.Med.Leg. 70-71: 31-47.

- Mata Fontanet, P. 1874. Tratado de Medicina y Cirugía Legal. Madrid. Bailly-Bailliere, ed.

- Miquel Feucht, M.J. \& J.D. Villalaín Blanco. 1996. El primer tiro de gracia: Estudio criminológico de un cráneo morisco. Rev Esp Med Leg . 76-77: 47-62.

- Muñoz, J.I., Linares-Iglesias, M., Suarez-Penaranda, J.M., Mayo, M., Miguens, X., Rodriguez-Calvo, M.S. \& L. Concheiro. 2001. Stature estimation from radiographically determined long bone length in a Spanish population sample. Journal of Forensic Sciences 46(2): 363-6.

- Muñoz Tuero, L.M., De Portugal Álvarez J. 1966. Aportación a la determinación de la edad en un cráneo. Anales de Medicina Forense de la Asociación Española de Médicos Forenses. VIII Jornadas Médico Forenses. Zaragoza.

- Muñoz Tuero, L.M., Moya Pueyo, V., \& J.D.Villalaín Blanco. 1972. Aportación a las muertes por proyectiles de arma corta de fuego. Estudio de restos óseos. Anales de Medicina Forense. Primera Reunión Hispanonorteamericana de Medicina Forense. PittsburgMadrid. 179-184.

- Muñoz Tuero, L.M. \& J. Díaz Domínguez. 1981. Aportación a las lesiones en restos óseos. Rev Esp Med Leg 26-27: 102-105.

- Naciones Unidas. Oficina de las Naciones Unidas en Viena. 1991. Centro de Desarrollo Social y Asuntos Humanitarios. Manual sobre la Prevención e Investigación Eficaces de las Ejecuciones Extralegales, Arbitrarias o Sumarias. Nueva York.

- Olóriz Aguilera, F. 1884. Recolección de Cráneos para Estudios Antropológicos. Granada. Librería de Paulino Ventura Sabatel.

- Olóriz Aguilera, F. 1894. Distribución geográfica del índice cefálico en España deducida del examen de 8.368 varones adultos. Memoria presentada al Congreso Geográfico Hispano-PortuguésAmericano en sesión de 19 de octubre de 1892. Madrid. Imp. del Memorial de Ingenieros.

- Olóriz Aguilera, F. 1896. La talla humana en España: discursos leídos en la Real Academia de Medicina el día 24 de mayo de 1896 para la recepción pública del académico electo. Madrid. Imp. y Libr. de Nicolás Moya.

- Olóriz Aguilera, F.1908. Dactiloscopia. Madrid. Imprenta de Eduardo Arias.
- Olóriz Aguilera, F. 1909. Guía para extender la tarjeta de identidad según las lecciones dadas en la Escuela de Policia de Madrid. Madrid. Imprenta de los Hijos de M.G. Hernández.

- Olóriz Aguilera, F. 1910. Experimentos de identificación monodactilar en la Universidad de Madrid. Madrid. Hijos de Reus.

- Olóriz Aguilera, F. 191I. Manuel Pour L'Identification des Délinquants de Madrid. Bruxelles. Ferdinand Larcier.

- Peiró P.M. \& J. Rodrigo. I844. Elementos de Medicina y Cirugía Legal Arreglados a la Legislación Española. Zaragoza. Imprenta de Mariano Peiró.

- Pérez de Petinto Bertomeu, M. 1952. Valor jurídico de la identificación de reliquias. Rev.Med.Leg. 72-73: 122-158.

- Pérez de Petinto Bertomeu, M. 1980. La estatura de una persona en vida deducida por la proporcionalidad ósea de sus restos esqueletizados. Rev Esp Med Leg 24-25: 64-71.

- Piga y Pascual, A. 1928. Medicina Legal de Urgencia. (La Autopsia Judicial). Madrid. Mercurio ed.

- Piga y Pascual, A. 1935. Manual Teorico-práctico de Medicina Legal. Madrid. Instituto Reus.

- Porter, G. \& G. Doran. 2000. An anatomical and photographic technique for forensic facial identification. Forensic Science International II4(2): 97-105.

- Prieto, J.L. 1996. Identificación dental. Técnicas radiológicas. Rev Esp Med Leg 76-77: 71-83.

- Prieto, J.L. \& J.M. Abenza. 1998. Métodos para valorar la edad en el adolescente. Rev Esp Med Leg. 84-85: 45-50.

- Prieto, J.L., Sánchez, J.A., Magaña, C., Roselló, J. \& A. Gremo. 200I. Curso Básico de Antropología Forense. Boletín Galego de Medicina Legal e Forense. Ponencias del curso organizado por la Asociación Gallega de Médicos Forenses.10.

- Prieto, J.L. 200I. Sistemática de la Recuperación de Restos Cadavéricos. Boletín Galego de Medicina Legal e Forense 10: 5.

- Prieto, J.L., Magaña, C., \& D.H. Ubelaker. 2004. Interpretation of postmortem change in cadavers in Spain. Journal of Forensic Sciences 49(5): 918-23.

- Prieto, J.L., Barberia, E., Ortega, R. \& C. Magaña. 2005. Evaluation of chronological age based on third molar development in the Spanish population. International Journal of Legal Medicine II9(6):349-54.

- Prieto J.L, Magaña, C., Bedate, A., Segura, L., Tortosa, C., Conejero, J., Abenza, J.M., Mariscal de Gante, M.C. \& B. Perea. 2005 Los atentados de Madrid del II de Marzo de 2004. Organización de las tareas médico-forenses en el pabellón NNo. 6 de IFEMA. Boletín Galego de Medicina Legal e Forense 14: 19-26.

- Prieto J.L. 2007. Stab wounds. The contribution of forensic anthropology. A case study, in Brickley, M.B. \& R. Ferlini (eds.) Forensic Anthropology: Case Studies from Europe: 19-37. Springfield, IL: Charles C. Thomas.

- Reverte Coma, J.M. 1997. Historia del Museo de Antropología Forense, Paleopatología y Criminología de la Escuela de Medicina Legal de la Universidad Complutense. Anales de la Real Academia Nacional de Medicina. II4(4): 865-82. 
- Ramírez Álava, M.A., Carnicero Giménez de Azcárate, M.A., Baigorri Soler, M.C. \& F. Etxeberría Gabilondo. 2000. El valor de la patología ósea en la identificación personal, a propósito de un caso con espondilitis anquilosante. Cuadernos de Medicina Forense. 22: 53-58.

- Reverte, J.M. 199I. Antropología Forense. Madrid. Ministerio de Justicia.

- Río Muñoz, P.A. \& J.A. del, Sánchez Sánchez. 1997. Discriminación sexual en la séptima vértebra cervical mediante el análisis de imagen. Rev Esp Med Leg . 80-81: 49-54.

- Río Muñoz, P.A. del, Sánchez Sánchez, J.A. \& J.L. Prieto Carrero. 2000. Determinación del sexo mediante el análisis de imagen en el atlas. Cuadernos de Medicina Forense. 22: 45-52.

- Río Muñoz, P.A. del, Sánchez Sánchez, J.A. \& J.L. Prieto Carrero. 200I. Estimación del sexo en la mandíbula mediante funciones discriminantes. Cuadernos de Medicina Forense. 26: 21-28.

- Rodes Lloret, F. \& J.B. Martí Lloret. 200l. Antropología Criminológica. Alicante. Universidad Miguel Hernández.

- Rodes, F. 2004. Foramen esternal vs orificio por proyectil de arma de fuego. Cuadernos de Medicina Forense 35: 71-74.

- Rodes Lloret, F., Giner Alberola, S., Pastor Bravo, M., Martí Lloret, J.B. \& E. Dorado Fernández. 2004. Herida craneal por arma de fuego en forma de "orificio en herradura". A propósito de un caso. Boletín Galego de Medicina Legal e Forense 13: 59-61.

- Rodríguez, J.V. 2004. La Antropología Forense en la Identificación Humana. Bogotá. Universidad Nacional de Colombia.

- Romero Palanco, J.L., Torres Ortiz, M.A. \& E. Vila Lopez. 1980. Estudio de la flora en cadáveres momificados. Rev. Esp. . Med. Leg. 24-25: 199-202.

- Royo-Villanova Morales R. 1952. Lecciones de Medicina Legal. Madrid. Marbán eds.

Salillas, R. 1888. La antropología en el derecho penal : tema de discusión en la Sección de Ciencias Exactas, Físicas y Naturales del Ateneo Científico, Literario y Artístico de Madrid para el curso de 1888-89. Madrid. Imprenta de la Revista de Legislación y Jurisprudencia.

- Salillas, R. 1908. Sentido y tendencia de las últimas reformas en criminología. Madrid. Asociación Española para el Progreso de las Ciencias. Imprenta de Eduardo Arias.

- Sanabria, C. 2004. Antropología Forense y la Investigación Médico-Legal de las Muertes. Bogotá. Facultad de Investigación Criminal.
- Sánchez, J.A. 1996. Antropología forense. Revisión histórica y perspectivas actuales. Rev Esp Med Leg 76-77: 63-70.

- Sánchez, J.A. 1997. Desastres de masas: legislación y tipo de accidentes. Rev Esp Med Leg. 78-79: 5I-56.

- Schiwy-Bochat, K.H., Riepert, T. \& M.A. Rothschild. 2004. The contribution of forensic medicine to forensic anthropology in Germanspeaking countries. Forensic Science International I44: 255-258.

- Serrano Cepedano, F. 1982. Acción del tiempo y la naturaleza sobre restos humanos. Rev Esp Med Leg 30-31: 79-82.

- Stewart, T.D. 1979. Essentials of Forensic Anthropology: Especially as Developed in the United States. Springfield. Charles C. Thomas. - Trancho,G.J, Robledo, B. \& I. López Bueis. 1997. Anthropological investigations in Spain. Madrid. University of Complutense.

- Trancho, G.J., Robledo, B., López Bueis, I. \& J A. Sánchez. 1997. Sexual determination of the femur using discriminant functions. Analysis of a Spanish population of known sex and age. Journal of Forensic Sciences 42(2):181-185.

- Ubelaker, D.H. 1996. Skeletons Testify: Anthropology in Forensic Science. Yearbook of Physical Anthropology 39: 229-244.

- Ubelaker, D.H. 1999. Human Skeletal Remains: Excavation, Analysis, Interpretation. Washington. Taraxacum.

- Valenzuela, A., Martin-de las Heras, S., Marques, T., Exposito, N. \& J.M. Bohoyo 2000. The application of dental methods of identification to human burn victims in a mass disaster. International Journal of Legal Medicine II3(4): 236-9.

- Ventura, F., Zacheo, To, Luck, To, \& A. Shovel. 2004. Computerised anthropomorphometric analysis of images: case report. Forensic Science International I46 Suppl: S2II-3.

- Vibert, Ch. 1916. Manual de Medicina Legal y Toxicología Clínica y Médico-Legal. Traducción castellana enriquecida con notas y referencias a la legislación española vigente por Manuel Saforcada. Barcelona. Hijos de J. Espasa.

- Villalaín Blanco, J.D. \& J. Buján Varela. 198I. Estudio de un cuerpo momificado hallado en Colmenar Viejo (Madrid). Rev Esp Med Leg 26-27: 56-58.

- Villalaín Blanco, J.D. \& M.T. Ramos Almazán. 198I. Consideraciones médico legales en relación al cuerpo momificado de Colmenar Viejo. Rev Esp Med Leg 26-27: 68-80.

- Villalaín Blanco, J.D. \& F.J. Puchalt Fortea. 2000. Identificación Antropológica Policial y Forense. Valencia. Tirant Lo Blanch. 